\title{
Sustainable Development Concepts as a Response to Expectations of Modern Business Management
}

\author{
D. V. Filatova ${ }^{1,2}$, and R.V. Fedorenko ${ }^{3, *}$ \\ *Corresponding author: fedorenko083@yandex.ru. \\ ${ }^{1}$ CHArt, EPHE, Paris, France \\ ${ }^{2}$ Belarusian State University of Informatics and Radioelectronics, Minsk, Belarus \\ ${ }^{3}$ Samara State University of Economics, Samara, Russian Federation
}

\begin{abstract}
The interactions among individual economic agents require some regulations to hedge the risk of business activity. This paper examines the general equilibrium theory, backgrounds of system analysis and optimal control to show that these regulations present the mechanism of balanced management of economic processes at various economic level (enterprise, region, country). The study shows that sustainable development is self-regulatory and adaptive effort to remain on the market under the condition of reliable cooperation. Furthermore, by investigating the hierarchy of the business management systems, the conceptual and mathematical models of the mechanism for sustainable development are built and illustrated.
\end{abstract}

Keywords: sustainable development, management, region, integration.

\section{Introduction}

The ongoing process of globalization demands searching for new, effective methods of business management. Independent actions undertaken by business entities in order to reduce costs or improve their profitability often prove to be insufficient. Nowadays, establishing cooperation with other enterprises or even regions is a way of increasing their strength and achieving market success. The legal framework of establishing and operating agreements showed that those vertical agreements these days are one of the most beneficial form of cooperation between partners. An opportunity for better coordination between the involved entities is their main advantage, which results in improving the economic efficiency of business activities. The European Commission presents a similar viewpoint, claiming that these agreements may contribute in particular to lowering the transaction and distribution costs as well as to optimizing the levels of sales and investment of cooperating parties [1]. Thus further attention will be focused only on enterprises or regions cooperating on vertical principles (they will be referred to as "a group"). In order for the undertaken cooperation to satisfy all the participants of the agreement and to provide the intended benefits over a longer period of time, its development must be balanced. This means considering the interests of all parties involved. Various methods are known to reconcile the interests of cooperating enterprises. Determining their so-called sustainable development is one of these methods. There exist various definitions of sustainable development [2, 3]. Most often it is defined as "development that meets the prevailing needs of society, without diminishing opportunities for development of future generations" [4]. This definition is too broad and does not make it possible to implement strict methods of managing complex economic structure. It is then necessary to indicate the determinants of such development. Therefore the aim of the paper is to determine the mechanism of sustainable business management of a group as a complex economic entity.

\section{Methods}

The study will be conducted using theoretical and empirical methods. The general equilibrium theory, the concepts of system analysis and optimal control will be applied to achieve the objective of the paper. This will allow us to indicate the determinants of sustainable business management, build a dynamic mathematical cooperation model in the form of optimal control problem.

\section{Concepts of sustainable management}

\subsection{Determinants and definition}

Let us consider a business situation of sustainable management of an enterprise (it can be also a region). The solution to a problem can be obtained by means of the system analysis. That is to say, the enterprise (on micro-level) or region (on macro-level), as the object of investigation, has to be discussed as a complex system [5].

Intuitively, the complex system can be defined as a hierarchical multilevel structure of interconnected homogeneous or heterogeneous elements which constitute an ordered and single whole. The elements of the system constitute the internal environment (each enterprise is a subsystem), the system itself is surrounded by an uncertain external 
environment (enterprise faces competition, whose behavior is difficult to forecast). Apart from that, the system must have the following features, including: integrality and emergence, organization (having structure and functions), functionality (showing certain properties when affecting the external environment and pursuing a goal), development (irreversible, targeted, regular change of the internal environment, which results in a new quality), self-regulation (striving to maintain the state of internal balance despite the changes in the external environment), endurance (suppressing harmful effects of the external environment), adaptation (ability to change behavior in order to maintain or improve the existing features or acquire new ones in an unspecified environment), reliability (ability to maintain structure despite the incapacity for work of distinct elements of the system).

The determinants of group's sustainability can be distinguished when analyzing the above-mentioned properties. They include: self-regulation, endurance, adaptation, reliability. These properties indicate the possibility of achieving business objectives not only for a group but also for its each member, as well as long-term cooperation regardless of the harmful activities of the competition. Therefore group sustainability will be understood as such self-regulatory and adaptive efforts to remain on the market under the condition of reliable cooperation, which results in development aimed at achieving business objectives.

\subsection{Decomposition and integration of the business management systems}

Achieving sustainability requires the implementation of appropriate methods of group management or coordination. It should be mentioned that within the framework of vertical agreements the interests of each party are equally important, hierarchy of enterprises (regions) and favoring the interests of one partner is not allowed. The management method is to be formulated as a joint program, e.g. manufacturing. This requires a quick exchange of the most important information that is usually subject of a cooperation agreement. This exchange is achieved through appropriate decomposition of the management systems of each enterprise and further integration of selected subsystems into a coherent group management (sometimes only informative). The following items are characteristic of each enterprise: hierarchy of management (management levels - strategic, tactical or operational management), and functions or subsystems of management (planning, controlling, motivation, organization, coordination) [6]. The five management subsystems can be considered as the decomposition of management system of each enterprise. Integration demands analyzing the functions of particular subsystems and understanding their hierarchy [7].

Let us consider this problem exclusively from the perspective of information flows. The elements of the planning subsystem include information system containing a database along with the database management system, the procedures for collecting and analyzing the information acquired and the system of interrelated plan of delivery, production, marketing, etc. at each management tier.

The formation of an integrated planning subsystem consists of four stages. The first stage consists in developing the parameters of the information system and thus determining the required amount of information, its scope, direction, content and specificity level, as well as defining the methods of its storage and access. The second and third stage consists respectively in developing the procedures of collecting and analyzing information. The last, fourth stage consist in developing procedures of devising plans, developing entry forms, time frames and appointing individuals responsible for formation, coordination and approval. The elements of the control subsystem as part of the information system include: a system of plans, with a system of target indicators, a system of standards for the operations of entities; "management indicators". On the other hand the procedures of subsystem control involve: monitoring the current operations of an enterprise; assessment/measurement of the business entities perform assessment/measurement of the implementation of plans; transmission and dissemination of information. It should be noted that the parameters of the control subsystem are entirely dependent on the parameters of the planning subsystem, therefore the control subsystem should be formed after the planning subsystem has been created. The formation of this integrated subsystem involves defining a set of indicators and determining their quantitative values, as well as developing the procedures for monitoring, assessment/measurement, transmission and dissemination of information. The staff motivation subsystem depends on the two previous subsystems, and it is created to meet the strategic and operational goals of enterprises. The system of indicators used to assess the performance of various entities and the system of indicators of employee performance are the elements of the information system here. The procedures include assessing and motivating employees. The elements of the subsystem of forming the organizational structure include the description of the functions performed in the enterprise, powers and responsibilities of the executive entities and the organization scheme of the enterprise. The organizational structure itself is created in several stages, namely: defining executive functions in the enterprise and executive entities implementing the functions, distribution of the functions by links; organizational analysis; defining professional responsibilities, devising the "Statute of the organizational structure". The key elements of the coordination subsystem, whose formation is based on the principles of operation of the four previous subsystems, include responsibility and authority understood as a limited right to use the organization's resources and perform delegated tasks. Management procedures imply clear decision-making methods [8]

It results, inter alia, from the fact that the powers are limited to plans, procedures, rules and verbal orders of managers, as well as environmental factors. In the case of the organizational structure formation subsystem the integration involves the organization of the strategic management process, organization and formalization of the management process as well as the implementation of new management procedures. After a detailed analysis of each management subsystem, allowing for the management tier, subsystems can be incorporated into an integrated management system (IMS) which fully reflects the needs of all stakeholders: employees, customers and the public. 
This integration enables effective (and consequently, sustainable) management of the region, enterprise, projects, processes, etc. as it is easy to track the performance of individual tasks and information flows [9] The advantage of the IMS is not only simplicity, transparency, uniformity and explicitness, but also the capability of parallel existence with other business management systems. One can assume that IMS is one of the solutions to sustainable group management. This means that enterprises cooperating in a group can agree on selected model (a kind of mechanism) to ensure sustainable development of the group without specifying activities that will lead to achieving business objectives. Summing up, the integration of the group management systems can be reduced to the integration of planning subsystems (at the strategic level) on the basis of the selected model using the determinants of sustainable development.

\section{The mechanism for sustainable management}

Let us consider a certain product (stock) whose overall production size at time $t \in\left[t_{0}, t_{1}\right] \subset \mathrm{R}_{+}$is a function $x:\left[t_{0}, t_{1}\right] \rightarrow \mathrm{R}_{+}$. Denote by $\theta_{1} \in \mathrm{R}_{+}$the intrinsic growth rate and by $\theta_{2} \in \mathrm{R}_{+}$the warehouse optimal capacity. Here we assume that the production growth rate takes the form of an ordinary differential equation

$$
\frac{d x(t)}{d t}=f(x(t)), X\left(t_{0}\right)=x_{0}, t \in\left[t_{0}, t_{1}\right],
$$

where $x_{0}$ is the initial value of the stock and the $\mathrm{C}^{2}$ function $f: \mathrm{R}_{+} \rightarrow \mathrm{R}_{+}$depends on $\theta_{1}$ and $\theta_{2}$ and meets the following conditions, namely: $f$ is concave $(\mathrm{c} 1), f(0)=0(\mathrm{c} 2)$, and $f\left(\theta_{2}\right)=0(\mathrm{c} 3)$. There is no loss of generality to assume that $f$ is a $\mathrm{C}^{2}$ function. Combining (c1) with (c2) and (c3) we get that the function $f$ has a unique maximum, say $x_{\max }$, as soon as $f$ is not the null function on the set $\left(0, \theta_{2}\right)$. The choice of $f$ function as a limited growth one

$$
f_{0}(x)=\theta_{1} x\left(1-\frac{x}{\theta_{2}}\right), x \geq 0,
$$

gives the following solution of (1)

$$
x(t)=\frac{\theta_{2}}{1+a \cdot \exp \left(-\theta_{1} t\right)}, t \in\left[t_{0}, t_{1}\right],
$$

where $a=\frac{\theta_{2}-x_{0}}{x_{0}}$ is a real number. The solution (3) has the following explanation, namely for any $t \in\left[t_{0}, t_{1}\right]$ whenever $x(t)<\theta_{2}$ the overall production will increase, if $x(t)>\theta_{2}$, it will decrease, and if $x(t)=\theta_{2}$, it will stay in a stable equilibrium. This conclusion can be used as a measure of the sustained management. We assume that the product is continuously consumed at a rate $u(t), u:\left[t_{0}, t_{1}\right] \rightarrow \mathrm{R}_{+}$. This allows on the rewriting (1) as follows:

$$
\frac{d x(t)}{d t}=f(x(t))-u(t), x\left(t_{0}\right)=x_{0}, t \in\left[t_{0}, t_{1}\right],
$$

where the $\mathrm{C}^{2}$ function $f$ fulfills the conditions (c1)- (c3).

Let us study the situation when the function $u$ is constant. As it is possible to see for any $t \in\left[t_{0}, t_{1}\right]$ whenever $0<u<f\left(x_{\max }\right)$ the curve of the function $f$ and the strait line $y=u$ intersect at the points $\left(x_{1}, u\right)$ and $\left(x_{2}, u\right)$, where $0<x_{1}<x_{\max }<x_{2}<\theta_{2}$. So, for constant consumption rate $u$ whenever $u<\max f(X)$, the equation (4) has one stable and one unstable equilibrium, however, whenever $u>\max f(X)$, there are no equilibria, if $u=\max f(X)$ a single semi-stable equilibrium exists at $X=x_{M S Y}$. The point $x_{M S Y}$ is referred to maximum sustainable yield and can be used as a performance indicator. Applying the same reasoning, we can show that the sustainable consumption is given by

$$
u(t)= \begin{cases}u_{\max } & \text { if } x(t)>x_{M S Y}, \\ f\left(x_{M S Y}\right) & \text { if } x(t)=x_{M S Y}, \\ 0 & \text { if } x(t)<x_{M S Y} .\end{cases}
$$

In the sequel we will focus on the following situation. The profit of economic activity related to the product is some function $\pi(x, t)$. Taking into account the value of time as the standard economic mechanism, we introduce the discount factor:

$$
\rho(t)=\exp \left(-\int_{t_{0}}^{t} \delta(\tau) d \tau\right)
$$


where $\delta(\cdot) \in \mathrm{R}_{+}$is a time-varying discount rate. The optimal sustainable consumption problem can be formulated as the following:

$$
\max _{u} \int_{t_{0}}^{t_{1}} \rho(t) \pi(t) u(t) d t
$$

subjected to (4) and

$$
0 \leq u(t) \leq u_{\max } .
$$

The task (4) - (6) is a singular optimal control problem, which solution is well-known and can be obtained by the Pontryagin's Maximum Principle. Therefore, referring to [10] and omitting technical details, we write the Hamiltonian

$$
\mathrm{H}=\rho(t) \pi(x, t) u(t)+\psi(t)(f(x)-u(t)),
$$

where $\psi(t)$ is the adjoint variable. Solving the task (4) - (6) we get for the consumption the bang-bang control

$$
u(t)= \begin{cases}u_{\max } & \text { if } \psi(t)<\rho(t) \pi(x(t), t), \\ 0 & \text { if } \psi(t)>\rho(t) \pi(x(t), t),\end{cases}
$$

and the singular control $u(t)=u^{S}(t)=\left(x^{*}(t)\right)-\frac{d x^{*}}{d t}$ for $\psi(t)=\rho(t) \pi(x(t), t)$ over $t \in\left[t_{0}, t_{1}\right]$, where the singular path $x^{*}(t)$ of (4) refers to the optimal sustainable production. As it is possible to notice from Fig. 1, the initial production is reduced by bag-bang control to the singular path $x^{*}(t)$, which can be calculated from the current changes of the economic parameters. To conclude, this solution is the mechanism for the sustainable management of the productionconsumption relations.

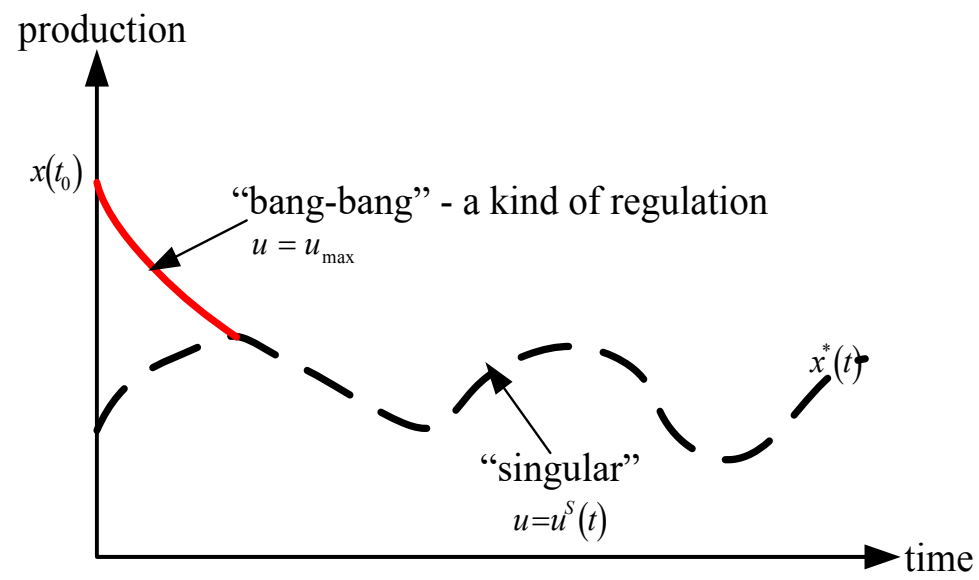

Fig. 1. The mechanism of sustainable management of the production process Source: Authors.

\section{Conclusion}

The ongoing globalization processes and the complexity of the interactions among individual economic agents require some regulations in order to achieve a kind of sustainable development. These regulations are considered as the mechanism of balanced management of economic processes at the level of enterprises and regions. The proposed balanced management mechanism has the potential for implementation at various economic levels from micro to mega one [11]. Obviously, the application of this mechanism for the decision-making will allow an enterprise or a region to maximize profits from economic activity by successfully integrating into the existing demand-supply economic system of a higher level. In some future research, the proposed approach can be considered as one of the tools for developing strategies for international economic cooperation at the level of states or interstate associations.

\section{Acknowledgment}

The reported study was funded by RFBR and FRLC according to the research project № 19-510-23001. 


\section{References}

1. W.V. van Themaat, B. Reuder, European competition law. A case commentary (Elgar Commentaries series). 2nd Edition (Edward Elgar Pub, New York, 2018).

2. J. Markard, R. Raven, B. Truffer, Sustainability transitions: An emerging field of research and its prospects. Research Policy, 41(6), 955-967 (2012). DOI: 10.1016/j.respol.2012.02.013.

3. B.B. Hughes, P.D. Johnston, Sustainable futures: Policies for global development. Futures, 37(8), 813-831 (2005). DOI: 10.1016/j.futures.2005.01.017.

4. R.R. Shaker, The spatial distribution of development in Europe and its underlyning sustainability correlations. Applied Geography, 63, 304-314 (2015). DOI: 10.1016/j.apgeog.2015.07.009.

5. G.P. Farnell, A.J. Saddington, L.J. Lacey, A new systems engineering structured assurance methodology for complex systems. Reliability Engineering \& System Safety, 183, 298-310 (2019). DOI: 10.1016/j.ress.2018.11.024.

6. A.C.-L. Chian, Complex systems approach to economic dynamics (Springer, Berlin, 2007).

7. T. Kaizoji, Towards economics as a new complex system. The European Physical Journal Special Topics, 225(17-18), 3225-3230 (2016). DOI: 10.1140/epjst/e2016-60161-X.

8. A. Bergek, S. Jacobsson, B. Carlsson, S. Lindmark, A. Rickne, Analyzing the functional dynamics of technological innovation systems: A scheme of analysis. Research Policy, 37(3), 407-429 (2008). DOI: 10.1016/j.respol.2007.12.003.

9. C. Binz, B. Truffer, Global innovation systems - A conceptual framework for innovation dynamics in transnational contexts. Research Policy, 46(7), 1284-1298 (2017). DOI: 10.1016/j.respol.2017.05.012.

10. A.V. Dmitruk, On the development of Pontryagin's maximum principle in the works of A.Ya. Dubovitskii and A.A. Milyutin. Control and Cybernetics, 38(4A), 923-957 (2009).

11. S. Nambisan, M. Wright, M. Feldman, The digital transformation of innovation and entrepreneurship: Progress, challenges and key themes. Research Policy, 48(8), 103773 (2019). DOI: 10.1016/j.respol.2019.03.018. 